

$$
\begin{aligned}
& \text { رئيس التحريز } \\
& \text { أ.د/ أميرة أحمد يوسف سليمان } \\
& \text { عميدة كلية البنات للآداب والعلوم والتربية }
\end{aligned}
$$

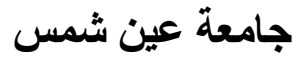

$$
\begin{aligned}
& \text { نائب رئيس التحرير } \\
& \text { أ.د// حنان محمد الثاعر } \\
& \text { وكيلة كلية البنات للار اسات العليا والبحوث } \\
& \text { جامعة عين شمس لاتس البن } \\
& \text { مدير التحريز } \\
& \text { أ.م.د/ أسماء فتحي توفيق }
\end{aligned}
$$

أستاذ علم النفس المساعد بقسم تربية الطقل

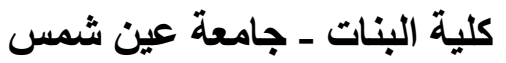

$$
\begin{aligned}
& \text { المحرر الفني } \\
& \text { أنور الهُي علي أحمد } \\
& \text { سكرتير التحرير } \\
& \text { نجوى إبراهيم عبا رباه عبد النبى لئي }
\end{aligned}
$$

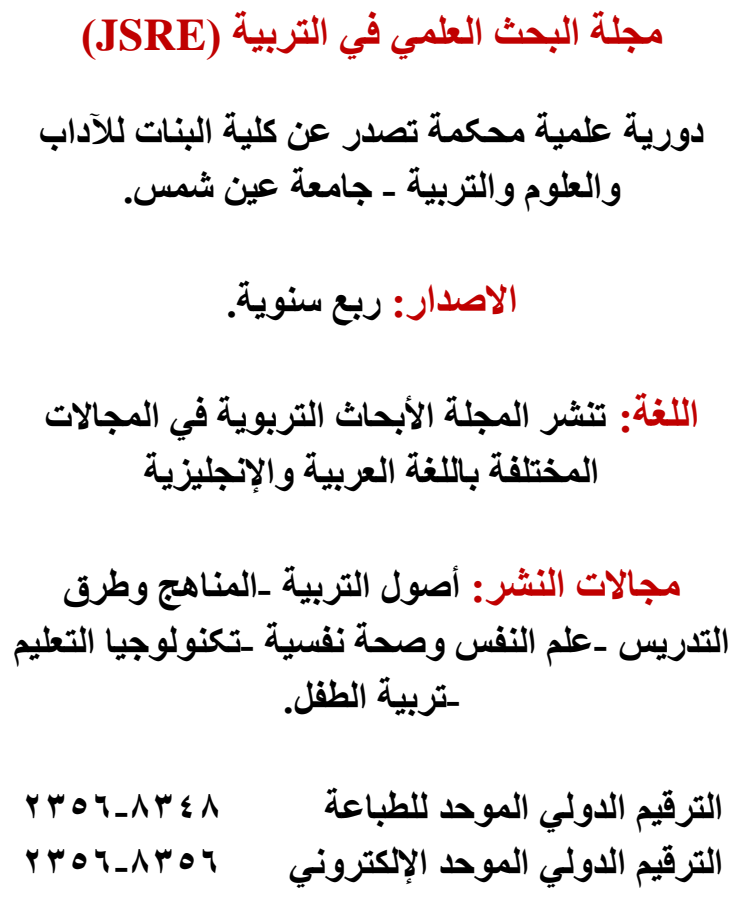




\title{
Utilisation des images sequentielles pour développer l'interprétation et la cohérence textuelle des futurs enseignants de la faculté de pédagogie à l'université de Minia et son effet sur la production écrite
}

\author{
Par/ Saif Elislam lofty Abdul Hakeem (*)
}

\section{Résumé}

La pratique des images séquentielles contribue automatiquement à la réalisation de l'enseignement/apprentissage de la langue, les apprenants sont amenés à ordonner les images d'un récit ou à inventer une histoire à travers des images séquentielles.

Inventer une histoire à travers les images n'est pas un exercice facile, d'abord la lecture (l'interprétation) des images, ensuite trouver une relation logique entre ces images. Choisir les images doit être judicieux, et la présence de ce lui qui enseigne est nécessaire afin de guider les apprenants, la temporalité se passe avec et par le langage et chaque étudiant raconte son histoire.

Pour bien interpréter une histoire à travers les images séquentielles, on met l'accent sur de sens dénoté (ce que l'on perçoit, reconnait et nomme), et de sens connoté (ce que l'on comprend, ce que l'on imagine).

La cohérence textuelle est une relation harmonieuse et une suite logique existantes entre les phrases et les différentes parties d'un texte, faisant de lui un tout.

Cette recherche s'est interessé au développement de quelques habiletés de l'interprétation et la cohérence textuelle a partir des images sequentielles,

le programme élabore a été appliqué pendant le $1^{\text {er }}$ semestre 2020/2021 aux futurs enseignants de la faculté de pédagogie à l'université de Minia. (20 étudiants), Cet échantillon se compose d'un seul groupe qui va étudier les modules proposés,

Le chercheur a utilisé la lecture des images séquentielles comme outil pour developper aux futurs enseignants quelques habiletés de la cohérence textuelle.

Les résultats de la recherche ont confirmé que le programme proposé a un effet positif sur la production écrite

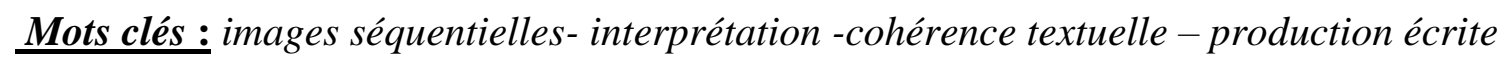

*Maître de conférences de méthodologie de FLE à la faculté de pédagogie de Minia Email:saifulislam_lotfy@yahoo.com

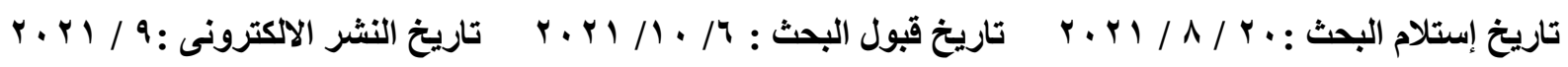




\section{Introduction :}

«... L'illustration est une image narrative, un art appliqué qui implique qu'elle a un sens. De nombreuses images présentent des compositions de couleurs et de formes qui n'ont à priori pas de sens. L'illustration possède un sens et s'applique à un texte, implicite ou réel. Même dans les albums sans texte, les images sont organisées en scénario et dans une volonté de donner du sens.

L'illustrateur est obligé de se référer à l'histoire pour donner du sens à ses images, Pour comprendre une illustration il faut acquérir des connaissances, des savoir-faire et savoir-être, maitriser le code et faire des inférences, intra illustration et inter illustrations. . » (Daniel Maja, 2004)

Tout d'abord, il y a des principes pour mettre les lecteurs des images séquentielles en réflexion :

1) il faut bien déterminer les images: choix des évènements connus de la part des lecteurs, dessins bien organisés lisibles, nombre suffisant de séquences.

2) mettre en considération que la succession logique de scènes nécessite de comprendre ce qui explique ces successions, les notions de causalité possible/impossible réversibilité/non réversibilité. Les mots de la logique utilisés par les apprenants: parce que, comme, alors, etc. constituent la marque de cet apprentissage

On doit s'intéresser à la mise en réflexion des étudiants au sujet du lien entre les images: on doit leur dire que c'est un problème à résoudre, les apprenants doivent essayer différentes solutions et en discuter à plusieurs. La temporalité se construit, elle ne s'enseigne pas. (B.Boishardy, 2008)

Il est important de interpréter les images séquentielles et d'élaborer un jugement au cours de l'apprentissage, les étudiants découvrent les textes cachés à ces images séquentielles, où l'implicite, la nature des visées, les références intertextuelles et les contextes culturels de production doivent être repérés et compris.

«...Lorsque les images sont présentées sans aucun texte aux récepteurs, ces derniers éprouvent la nécessité d'ajouter un message linguistique. Ils imaginent par exemple des slogans ou des légendes pour les images; ils leur attribuent des titres. En s'adressant à une cible, ils sont amenés à jouer le rôle de l'auteur ou du publicitaire. » (Catherine Muller, 2013: 128)

On trouve que les apprenants égyptiens qui étudient la langue française en tant que deuxième étrangère souffrent de l'insuffisance de leur habileté interprétative, il parait que l'habitude de la lecture des images séquentielles en langue arabe chez les étudiants n'est pas automatiquement transférable à la lecture d'images séquentielles en français. 
Pour bien interpréter les images séquentielles à travers les images, on trouve qu'il y a plusieurs séquences textuelles : la séquence narrative, la séquence descriptive la séquence explicative, et la séquence argumentative.

Cette recherche a mis l'accent sur la séquence narrative qui est au service de la cohérence textuelle à l'intérieur d'un récit puisque tout récit repose sur le développement d'une intrigue qui est l'enchaînement logique des événements menant au dénouement.

Quand on raconte une histoire, il est facile d'en trouver le commencement, le milieu et la fin de l'histoire, les relations sémantiques entre les parties du texte liées à ces trois éléments de l'histoire ont pour but de progresser la cohérence.

L'organisation narrative de la succession des événements de l'histoire séquentielle

dépend de l'habilité de l'apprenant à distinguer les deux moments

principales de l'histoire, qui coïncide avec la première image et la derrière.

Cette recherche a confirmé le manque de la cohérence des textes produits par les futurs enseignants, le chercheur a observé dans la plupart de grilles de corrections de la production écrite le critère suivant : «le texte doit être cohérent ».

Parmi les fautes de la cohérence textuelle les plus habituelles dans les copies des futurs enseignants de la faculté de pédagogie de Minia on peut trouver :

a) Les signes de ponctuation, qui servent à séparer les phrases ou les mots, mais aussi à marquer des nuances dans la pensée, des relations logiques entres les phrases ; en effet, lorsque les futurs enseignants n'utilisent pas de signes de ponctuation, les phrases deviennent trop longues et leurs productions sont souvent incompréhensibles.

b) Les « qui » et les « que ». Il s'agit, bien sûr, d'un problème linguistique, mais qui gêne la cohérence du texte lorsque ces pronoms sont mal utilisés.

c) Les organisateurs du discours, qui sont souvent mélangés ou absents, dans une relation. Par exemple :

Premièrement... Ensuite... Troisièmement (Corrigé: Premièrement...Deuxièmement...Tro isièmement)

D'un autre côté... / D'autre part...(on ne peut pas les utiliser si on n'a pas utilisé avant D'un côté... / D'une part...

Les connecteurs logiques servent à établir des relations entre deux idées, deux faits et expriment la cause, la conséquence, l'opposition, l'addition et la reformulation.

Pour écrire un bon texte, il ne suffit pas d'avoir des idées mais il faut appliquer des règles concernant la cohérence et la cohésion textuelle afin d'assurer un texte de qualité et de clarté 
chez le futur enseignant, et également pour obtenir a une correction plus efficace de la part de l'enseignant.

La cohésion du discours repose sur les relations sémantiques, et plus largement linguistique qu'il instaure entre les phrases, enchainement syntaxique, la reprise anaphorique, mais aussi les récurrences thématique ou référentielles et l'organisation temporelle des faits évoqués donne au texte une forte dimension cohésive.

L'étude de Emmanuelle Beaulieu HANDFIELD (2008) a essayé de remédier la rupture des cohérences dans les écrits de étudiants universitaires francophones, dans cette étude le chercheur a analysé 75 textes rédigés par des étudiants au baccalauréat, en enseignement éprouvant des difficultés en français écrit, à l'aide de grilles d'analyse de la cohérence textuelle élaborées par Lemonnier et Gagnon, Les résultats indiquent que les ruptures de cohérence sont causées par des énoncés dont le lien avec le contexte parait peu ou pas pertinent, et l'usage inadéquat de connecteurs ; il y a également des énoncés contenant de l'information superflue ou hors texte.

On emploie la cohérence textuelle à travers un système de règles déterminées, ce qui fait qu'un texte est un texte et non une suite de phrases sans rapport.

Ce point est digne d'être étudié afin que les futurs enseignants soient capables de rédiger un texte cohére et intelligible.

Le rôle du professeur est de localiser et signaler les fautes de l'écriture incohérente les étudiants doivent ensuite les corriger.

La cohésion concerne plutôt la forme du texte et se manifeste à l'intérieur de la phrase (connecteurs spatiaux, temporels, argumentatifs...). La cohérence est de caractère global et concerne les champs lexicaux, les idées, les relations entre les passages ; cela veut dire que les idées doivent se suivre logiquement l'une à l'autre.

Christian Vandendrope (1995:9) a indiqué que les différents éléments du texte doivent porter sur un thème commun et qu'il soit organisé d'une façon à offrir une progression, il faut que le fil thématique soit facilement repérable et qu'il puisse être suivi d'une phrase à l'autre pour arriver au sens.

Dans cette étude le chercheur se base tout au long de la recherche de la cohérence et la cohésion textuelle qui exige la présence d'une relation logique entre les phrases et les paragraphes qui permettent l'organisation d'un texte et un pouvoir de sens.

En ce qui concerne l'enchainement des phrases à l'intérieur d'un texte en respectant le thème de cohésion, on a mis l'accent sur les rapports de liaison entre les éléments constitués d'assurer le suivi d'une phrase à l'autre. 
$\underline{\text { Pour que le texte soit cohérent, cinq principes doivent être mis en application }}$

1. L'unité de sujet : L'ensemble du texte doit être centré autour d'un seul qui sera développé à partir des phrases et des paragraphes, le texte doit suivre une ligne directrice clarifiée par le sujet qui en est à la base.

2. La reprise de l'information: Pour maintenir l'unité du sujet, certains mots ou groupes de mots vont servir à reprendre certains éléments déjà mentionnés. Ces substituts désignent une réalité que l'on a nommée précédemment dans le texte.

3. Les séquences textuelles: les informations doivent être présentées dans un ordre logique et précis. Chaque nouvelle phrase et chaque nouveau paragraphe doit apporter des informations supplémentaires sous des formes variées : définition, explication, exemple, réflexion, etc.

4. La cohérence des informations: dans les informations présentées, il ne doit pas y avoir de contradiction dans le temps ou dans les événements. Si deux paragraphes d'un texte amènent des informations qui se contredisent, le lecteur va avoir beaucoup de mal à comprendre le texte.

5. La constance du point de vue: Le point de vue, c'est la manière dont l'auteur ou le narrateur se présente dans un texte.

Charolles (2011) a déterminé les règles pour qu'un texte soit reconnu étant cohérent. Selon lui, il faut que ce texte :

- Comporte dans son développement linéaire des éléments à récurrence stricte. La répétition est nécessaire pour tisser des liens entre les phrases et les séquences structurantes d'un texte.

- Un texte doit apporter constamment des nouvelles informations qui font progresser le texte. Ainsi, les redondances affectent la cohérence du texte

- Ne manifeste pas de contradictions,

- Dénote des faits qui sont reliés par des relations de cause, de condition ou de conséquence, exprimées le plus souvent par des connecteurs

- La cohésion se limite à l'utilisation correcte des connecteurs grammaticaux et de l'articulation dans la phrase, ce qui permet un séquencement harmonieux des phrases et des paragraphes dans un texte.

Le chercheur a essayé à travers cette recherche de développer quelques habiletés de l'interprétation et de la cohérence textuelle à travers les images séquentielles aux futurs enseignants de la faculté de pédagogie à l'université de Minia. 


\section{Position du problème.}

Le problème de notre recherche s'est posé à travers :

- Les résultats de quelques-unes des recherches précédentes comme les études d'Helmi (2000), d'Abdel-Ghany (2003), d'El Kouche (2004), de Sabri (2005), qui ont affirmé que la plupart des étudiants à l'université sont incapables de : -

- produire une introduction cohérente de texte ;

- produire un développement cohérente de texte ;

- produire une conclusion cohérente de texte ;

- Veiller à la cohésion des parties de la phrase ;

- Enchaîner les idées de texte.

Ces études ont confirmé que le traitement des difficultés de la cohérence textuelle

constituent la base essentielle pour développer les compétences de la production écrite et les autres compétences de FLE relatives au code écrit de la langue chez les apprenants notamment les étudiants au cycle universitaire

Ces résultats confirment ce que la recherche actuelle a essayé d'atteindre.

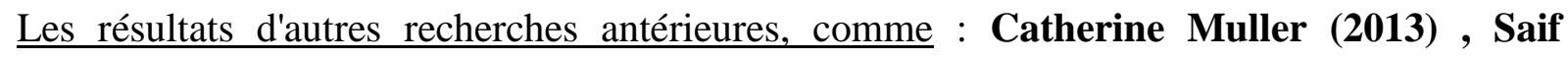
Elislam Lotfy (2020)

Ces études ont confirmé que les étudiants ont éprouvé aussi des difficultés lors de l'interprétation des images fixes par écrit

- Comme expérience personnelle: le chercheur a fait une interview avec un certain nombre de professeurs au département de la langue française (aux facultés des langues, des lettres, et de pédagogie à l'université de Minia)

et ils ont été d'accord sur l'incapacité des futurs enseignants à posséder quelques habilites de la production écrite (l'interprétation des images séquentielles et la cohérence textuelle de la langue française).

Ces étudiants ont éprouvé des difficultés lors de la rédaction d'un texte cohérent puisque, c'est la textualité qui fait qu'un texte est un bon texte et pas sa grammaticalité.

- Pour s'assurer de ces difficultés, on a mené une étude pilote destinée à un groupe des futurs enseignants (20 étudiants) au cours de l'année universitaire 2020/2021 au $1^{\text {er }}$ semestre et les résultats sont comme suit : 
La plupart des futurs enseignants de la faculté de pédagogie de Minia ne peuvent pas écrire un texte cohérent, ils ne respectent pas la structure du texte (introduction-développementconclusion), ils ne maitrisent pas le ménagement des transitions, ils ne mettent pas en considération de l'orthographe lexicale, la ponctuation et la mise en page (la majuscule, l'apostrophe le trait d'union, l'utilisation adéquate des abréviations etc.)

Ils ont également du mal à interpréter les images séquentielles par écrit, ils ne maitrisent pas le décodage des images séquentielles, ils ne peuvent pas décrire les interprétations possibles des images séquentielles.

La cause de ce problème est probablement due au manque de ces compétences chez les futurs enseignants. Ce manque des compétences peut être dû à l'utilisation de mauvaises stratégies d'enseignement que ne sont pas convenable à leurs styles d'apprentissage.

\section{Problématique de la recherche :}

Le problème de cette recherche s'est résumé dans quelques difficultés à la cohérence textuelle (incapacité à exprimer une pensée claire, à établir des liens et à écrire un texte structuré et cohérent), ce qui cause une faiblesse dans les compétences de la production écrite chez les futurs enseignants qui étudient le français comme (L2). Ces futurs enseignants de la faculté de pédagogie à l'université de Minia département de français souffrent aussi de l'insuffisance de leur compétence interprétative des images séquentielles par écrit d'une manière cohérente, le problème s'est résumé dans la question principale ci-dessous :

(Comment développer quelques habiletés de l'interprétation et la cohérence textuelle de la langue française chez les futurs enseignants de la faculté de pédagogie de Minia, section de français à travers l'utilisation des images séquentielles? et quel est l'effet de ce programme sur la production écrite des étudiants de l'échantillon ?)

\section{Cette question se divise en 4 sous questions :}

1) Quels sont les critères sur lesquels le chercheur s'appuie pour juger l'interprétation des images séquentielles chez les futurs enseignants de la faculté de pédagogie de Minia section de français?

2) Quels sont les critères sur lesquels le chercheur s'appuie pour juger la cohérence d'un texte de futurs enseignants?

3) Quel est le degré de maîtrise des habiletés de l'interprétation des images séquentielles et de la cohérence textuelle que possède les futurs enseignant?

4) Quel est l'effet de l'utilisation d'un programme proposé à la lueur des images séquentielles pour développer l'interprétation des images séquentielles et la cohérence textuelle chez les futurs enseignants faculté de pédagogie, section de français?

5) Quel est l'effet de l'utilisation de ce programme proposé sur la production écrite chez les futurs enseignants, section de français, faculté de pédagogie de Minia? 


\section{$\underline{\text { But de la recherche }}$}

Cette recherche vise essentiellement à atteindre les objectifs suivants :

1) Déterminer les habiletés de l'interprétation des images séquentielles nécessaire aux futurs enseignants faculté de pédagogie section de français.

2) Déterminer les habiletés de la cohérence textuelle nécessaire aux futurs enseignants faculté de pédagogie section de français

3) Associer un ensemble d'images séquentielles à un récit.

4) Identifier l'effet de ce programme proposé pour développer l'interprétation des images séquentielles et la cohérence textuelle

5) vérifier de l'efficacité de l'emploi d'un programme proposé pour développer l'interprétation des images séquentielles et la cohérence textuelle sur la production écrite chez les futurs enseignants, section du français, faculté de pédagogie de Minia.

\section{Importance de la recherche}

\section{La recherche en cours peut-être profitable pour :}

1) Les futurs enseignants de FLE au cycle universitaire dans le cadre de diagnostiquer scientifiquement les difficultés l'interprétation des images séquentielles et la cohérence textuelle chez eux. Les inspecteurs et les maîtres-enseignants de FLE dans la mesure de bénéficier des outils élaborés dans leur travail de formation.

2) Les didacticiens et les méthodologues de FLE, dans la mesure de bénéficier des exercices contenues dans le programme élaboré, pour remédier aux difficultés fréquentes de l'interprétation des images séquentielles et la cohérence textuelle.

\section{Méthodologie de la recherche.}

Pour atteindre les objectifs de la recherche en cours, le chercheur a suivi les méthodologies ci-dessous :

- La méthodologie analytique descriptive : le chercheur a étudié et a analysé les variables de la recherche, afin d'élaborer un cadre théorique cohérent et relatif à ces variables;

- La méthodologie expérimentale : le chercheur a mené une étude expérimentale (l'intervention), pour aider les futurs enseignants en FLE à surmonter leurs difficultés concernant l'interprétation des images séquentielles et la cohérence textuelle, l'échantillon de la recherche se compose d'un seul groupe qui va étudier les modules proposés.

- La méthodologie statistique : le chercheur a employé les techniques statistiques le programme statistique (SPSS), pour traiter les résultats etde les interpréter afin de s'assurer de l'efficacité de l'emploi des images séquentielle pour développer l'interprétation des images séquentielles et la cohérence textuelle. 


\section{$\underline{\text { L'échantillon de la recherche }}$}

- Un échantillon des futurs enseignants de la faculté de pédagogie à l'université de Minia, qui étudient la langue française comme deuxième langue étrangère.

On a choisi l'échantillon de $3{ }^{\text {ème }}$ année, Car ils ont besoin de bien maîtriser leur niveau linguistique après avoir acquis la base de la langue française pendant la première et la deuxième année. De plus, les mauvaises remarques qu'on a observé dans une étude pilote de certaines copies rédigées par ces étudiants, le chercheur a été convaincu que l'écriture représente un réel obstacle chez les futurs enseignants.

\section{Les outils et matériels de la recherche}

\section{Instruments de la recherche tels que :}

1- Une liste d'habiletés de l'interprétation des images séquentielles. Annexe (1)

2 - Une liste d'habiletés de la cohérence textuelle. Annexe (2)

3- Test de l'interprétation des images séquentielles et de la cohérence textuelle et le barème de correction de ce test. Annexe (3)

4 - Test de la production écrite et le barème de correction de ce test. Annexe (4)

\section{Matériaux de formation comme}

5 -un programme proposé qui comporte une série images séquentielles pour développer l'interprétation des images séquentielles ainsi que la cohérence textuelle.

Annexe (5)

6- un Guide pédagogique Annexe (6)

\section{$\underline{\text { Délimite de la recherche }}$}

- un échantillon des futurs enseignants de la $3^{\text {ème }}$ année de faculté de pédagogie à l'université de Minia qui étudient la langue française comme deuxième langue étrangère.

- Un programme proposé qui comporte 3 modules| : ces modules sont formulés selon les habiletés de l'interprétation des images séquentielles et la cohérence textuelle de la langue française,

- Le Programme d'entrainement proposé a commencé le 15 octobre 2020, et s'est terminé le 15 janvier $2021\left(1^{\mathrm{er}}\right.$ semestre $)$.

\section{Les hypothèses de la recherche}

\section{L'étude actuelle a essayé de vérifier des hypothèses suivantes :}

Le programme proposé a un effet positif pour améliorer les habiletés de l'interprétation des images séquentielles et de la cohérence textuelle de la langue française. 


\section{La recherche actuelle s'est proposée à vérifier $\mathrm{s}^{\prime}$ :}

1- Il existe une différence significative au seuil de $(0,01)$ entre la moyenne des notes des futurs enseignants de la faculté de pédagogie de Minia au (pré-post) test des habiletés de l'interprétation de la langue française, en faveur du post-test.

2- Il existe une différence significative au seuil de $(0,01)$ entre la moyenne des notes des futurs enseignants de la faculté de pédagogie de Minia au (pré-post) test d'habiletés de la cohérence textuelle de la langue française, en faveur du post-test.

3- Il existe une différence significative au seuil de $(0,01)$ entre la moyenne des notes des futurs enseignants de la faculté de pédagogie à la production écrite avant et après l'enseignement du programme en faveur du post test.

\section{Plan de la recherche}

Le plan de travail se constitue de deux volets :

\section{Une étude théorique abordant :}

- La lecture (l'interprétation) des images séquentielles.

- La cohérence textuelle de la langue française. Préciser la notion de cohérence et à élaborer graduellement une approche d'enseignement basée sur ces Principes

\section{Une étude pratique abordant :}

L'étude expérimentale :

- La préparation des instruments de la recherche.

- L'expérimentation.

- L'analyse et l'interprétation les résultats.

\section{Procédures de la recherche}

Pour répondre aux questions de cette recherche, le chercheur a suivi les démarches suivantes :

1)-Consulter des travaux et des études antérieures concernant les habiletés de la lecture (l'interprétation) des images séquentielles ainsi que la cohérence textuelle de la langue française et des images séquentielles

2)- Élaborer une liste d'habiletés de l'interprétation des images séquentielles de la langue française nécessaires aux futurs enseignants de la faculté de pédagogie de Minia.

3) -Élaborer une liste d'habiletés de la cohérence textuelle de la langue française

4)-Choisir 20 étudiants pour l'application de l'expérimentation.

5) -Élaborer un pré /post test de l'interprétation des images séquentielles et de la cohérence textuelle de la langue française aux futurs enseignants de la faculté de pédagogie de Minia. 
6) -Élaborer un programme proposé pour développer la cohérence textuelle de la langue française.

7) -Élaborer un pré /post test de la production écrite des futurs enseignants de la faculté de pédagogie de Minia.

8) -Appliquer le pré test de l'interprétation des images séquentielles et de la cohérence textuelle de la langue française aux futurs enseignants de la faculté de pédagogie.

9) -Appliquer le test de la production écrite aux étudiants de l'échantillon.

10) -Appliquer le programme proposé.

11) Appliquer le post test de l'interprétation des images séquentielles et de la cohérence textuelle de la langue française aux futurs enseignants de la faculté de pédagogie.

12) -Appliquer le post test de la production écrite aux étudiants de l'échantillon.

13) -Traiter statistiquement les résultats et Interpréter ces résultats.

14) proposer les recommandations et les suggestions.

\section{Terminologies de la recherche}

Programme : Descriptif écrit et détaillé des contenus de formation planifiés.

Il respecte une progression pédagogique liée aux objectifs de formation à atteindre.

\section{«Lexique de termes pédagogiques couramment utilisés dans le monde éducatif et de l'enseignement, 2006»}

Une image séquentielle : ou "histoires en images" sont un outil pédagogique et psychologique connu depuis longtemps des instituteurs et des personnels spécialisés (psychologues scolaires, rééducateurs, orthophonistes). Les images séquentielles se rattachent ainsi à une double tradition pédagogique et éditoriale : les "images à observer" ou "à raconter" d'un côté (lecture synoptique), les "planches d'images" ou "bandes dessinées" de l'autre (lecture linéaire).Les images sont soit présentées en paquet, soit alignées horizontalement dans le désordre. A partir de là, plusieurs modalités d'utilisation existent ou sont possibles :

- on peut demander à l'apprenant de mettre les images en ordre pour faire une histoire, de la raconter,

- on peut présenter l'histoire déjà rangée et demander à l'apprenant dela raconter ;

- on peut demander à l'apprenant de décrire chaque image (Proje-cretien Joëlle, Alves Christian 1989). 


\section{$\underline{\text { Le chercheur a proposé de définir les images séquentielles }}$}

Les images séquentielles sont un outil d'activité utilisé pour progresser les habiletés langagières (les compétences de repérage spatio-temporel, de repérer les liens logiques entre des images et de les remettre en ordre chronologique).

Interprétation : (C'est la façon de comprendre une chose)

«Dictionnaire du français ,2001»

\section{$\underline{\text { Le chercheur a proposé de définir Interprétation }}$}

C'est le déchiffrement et identification facilement les mots tout en comprenant relativement bien ce qu'on lit.

C'est la signification qu'on peut donner à une chose bien compris, bien expliquée.

La cohérence : se manifeste dans les liens qui se forment entre la microstructure « niveau des phrases » et la macrostructure « le thème développé d'une phrase à l'autre et qui forme un ensemble » la microstructure correspond à ce qu'on appelle la cohésion alors que la macrostructure représente la cohérence «Van Dick, 1984»

La cohérence du texte lui-même, sa logique et tout ce qui la constitue. C'est aussi développer la cohérence de la pensée : développer chez l'apprenant la capacité de faire des liens, de penser, de réfléchir le langage. «Jacqueline Lemieux, 1998 »

Le chercheur a proposé de définir La cohérence textuelle :

La cohérence textuelle c'est la facilite à développer une idée, à élaborer un texte ordonné et logique et à établir des relations entre les éléments du discours

\section{$\underline{\text { La production écrite }}$}

Une activité complexe qui met en jeu différentes opérations considérées comme récursives telles que

- la planification (opération qui vise à récupérer, à sélectionner et à organiser selon un plan les connaissances relatives au sujet contenues en mémoire à long terme) .

- la mise en texte (qui est la rédaction proprement dite et qui implique de faire les choix lexicaux adéquats, de gérer les aspects morphosyntaxiques et graphiques, de veiller à la cohérence et la cohésion du texte),

- la révision (opération qui a pour but d'améliorer le texte produit et qui comporte deux sous processus à savoir le repérage des lacunes et la mise au point ou correction «Bouchard, R. ,2000».

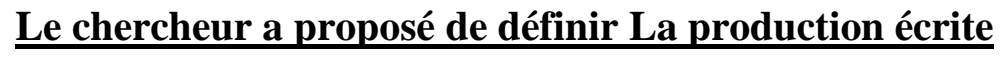

La production écrite c'est mettre en mots la pensée, et cela suppose de réunir les idées, de les organiser avec des outils de la langue écrite. 
À partir de la production écrite des futurs enseignants, l'étudiant peut être amené à évaluer comment il réussit à traduire sa pensée.

\section{Le Cadre théorique de la recherche}

Dans cette partie le chercheur a divisé le cadre théorique de la recherche en deux axes, la cohérence textuelle et la lecture des images séquentielles.

\section{La cohérence textuelle}

Le CECRL (le cadre européen commun de référence pour les langues) a définit l'acquisition des capacités discursives comme celle permettant à l'apprenant d'ordonner les phrases en séquences afin de produire des ensembles cohérents.

Selon le CECRL: dans l'apprentissage d'une langue étrangère, il est conseillée que l'apprenant commencera par de brefs énoncés d'une phrase seulement, en général aux niveaux supérieurs, le développement de la compétence discursive dont les composantes sont inventoriées dans cette section devient de plus en plus important, Les échelles suivantes viennent illustrer certains aspects de la compétence discursive :

- souplesse ;

- tours de parole ;

- développement thématique ;

- cohérence et cohésion. (CECRL, 2001, p. 96-98)

En ce qui concerne les deux compétences cohérence et cohésion (selon les compétences des apprenants de niveaux intermédiaires et avancés)

La cohésion concerne plutôt la forme du texte et se manifeste à l'intérieur de la phrase (connecteurs spatiaux, temporels, argumentatifs...). La cohérence est de caractère global et concerne les champs lexicaux, les idées, les relations entre les passages ; cela veut dire que les idées doivent se suivre logiquement l'une à l'autre.

\section{Les règles de la cohérence textuelle}

\section{«Pour bien réaliser la cohérence textuelle, il faut que le texte obéisse aux quatre règles} suivantes

(1) La progression de l'information veut que chaque nouvelle phrase apporte une information nouvelle par rapport à la logique et l'information précédente.

Dans un texte cohérent, la transition d'une idée à l'autre doit être assurée d'une manière claire. Cette transition logique est importante pour ne pas donner au lecteur l'impression que l'auteur saute d'un point à l'autre, sans lien logique.

\section{(2) La relation entre les paragraphes et les idées}


On trouve que dans les belles écritures, la fin de chaque paragraphe annonce le début du paragraphe suivant, alors il faut que chaque paragraphe apporte une idée nouvelle ou complémentaire de l'idée principale.

Il existe un rapport entre les paragraphes, ce rapport se caractérise par l'enchainement des idées d'un paragraphe à l'autre et aide à constituer un texte bien cohérent avec des faits relies entre eux.

\section{(3) Un champ lexical}

Le champ lexical comporte l'ensemble de vocabulaire qui compose le texte, ces vocabulaires doivent correspondre au sujet traité,

Dans un texte cohérent, les mots doivent être utilisés dans leurs justes titres, c'est-à-dire bien encadré dans leurs champs lexicaux pour avoir des phrases claires sans ambiguïté.

(4) La non-contradiction : c à d qu'il n'existe pas de contradiction entre les phrases et les paragraphes. La non-contradiction donne de la crédibilité au texte, en évitant d'opposer des informations clairement dites et non dites dans le texte.

Il existe deux types de contradiction

La contradiction énonciative : on doit éviter les modifications qui se passe soudain sans transition, comme le changement du temps de passe simple au passé compose ou le changement de personne du pronom je au pronom il.

La contradiction en plan référentiel : cette contradiction est reliée à la cohérence de plan référentiel, ex : le premier paragraphe parle de l'enseignement et l'autre tourne autour de la cuisine, ce changement du sujet appelé une digression inappropriée peut nuire à la cohérence textuelle. »

(Mohammed Alkhatib, 2012)

\section{$\underline{\text { L'interprétation des images séquentielles }}$}

Les images séquentielles se lisent d'une manière différente que la lecture d'un texte, en s'attachant à repérer plusieurs paramètres.

Le mode d'emploi des images séquentielles est simple : l'apprenant doit constituer une histoire à travers un nombre d'images variables, le minimum est deux images, et le maximum n'est pas limité puisque ça dépend de la motivation des apprenants et également leur habileté au traitement, souvent ils utilisent quatre images séquentielles.

Joëlle, Alves Christian, 1989)

$\underline{\text { Il y a plusieurs modalités d'utilisation qu'on peut utiliser à partir les histoires séquentielles : }}$

- Raconter une histoire à partir d'images.

- Mettre en ordre les différentes images d'une histoire (début, milieu, fin.). 
- Anticiper la fin d'une histoire.

Les histoires séquentielles permettent aux apprenants de développer des habiletés au niveau du langage, de la compréhension, de la logique et, de la notion du temps.

$\underline{\text { Parmi ces habiletés qui sont visées: }}$

La compréhension d'une histoire (étapes, ordre chronologique, etc.).

L'organisation des idées.

L'utilisation de indices (faire des liens et anticiper).

L'organisation de la tache placer les images (de gauche à droite).

L'ordre dans lequel les phrases sont présentées détermine le sens que on peut dégager, l'interprétation des images séquentielles qui est relative à l'image qui précède et à celle qui suit, le sens relié à l'entourage dans le texte et également dépendant du contexte ou de la situation d'énonciation.

L'interprétation des notions sémantiques entre les phrases à partir les deux éléments mentionnés détermine la cohérence textuelle.

Parfois, La fonction des indicateurs de temps précise le sens, et en permet l'interprétation ce qui établit le lien de cohérence entre deux phrases.

Pour bien interpréter un texte écrit concernant des images séquentielles, il faut que le texte développe un thème ou un sujet, l'interprétation des images séquentielles selon le thème qu'elle développe réalise la continuité logique et la cohérence.

Dans les textes narratifs le temps et le lieu où les évènements se déroulent détermineront la cohérence de certains énonces, la non considération du cadre spatio temporelle donne lieu à des énoncés qui n'ont pas de point d'ancrage dans la réalité et qui dans ce fait sont incohérents et évidemment, cela nous mène à une mauvaise interprétation du texte.

Il est important de choisir des grandes images, « lisibles », colorées et variées de manière à apprendre progressivement aux apprenants à «voir» les choses, à prélever des indices pertinents et à nommer avec précision.

On peut déduire que l'Interprétation des images séquentielles constitue la phase la plus importante et comporte deux étapes :

- Significations initiales et ultérieures : les significations initiales concernent le titre, la légende et le commentaire du producteur alors que les significations ultérieures concernent la signification qu'on peut attribuer à l'image actuellement. 
- Bilan et appréciations personnelles : à ce moment, le chercheur fait appel à sa subjectivité, dans le domaine de l'analyse de l'image afin de déduire un bilan général et de donner une appréciation subjective de l'image

Les réactions interprétatives des récepteurs constituent un prolongement de leurs interrogations sur les motivations de l'auteur. Il s'agit d'un phénomène par lequel les spectateurs attribuent un sens caché ou figuré aux images au-delà du sens littéral qu'ils perçoivent.

Cette réaction s'appuie sur une seconde lecture de l'œuvre dans laquelle les connotations et la polysémie apparaissent. Ces réactions interprétatives sont introduites par des verbes : «ça représente », «ça symbolise », « ça veut dire ». De telles expressions permettent aux apprenants d'expliciter le sens second qu'ils imaginent et de faire disparaître peu à peu le sens littéral initialement perçu.

Pour avoir les différentes interprétations des personnes qui observent l'image, les

détails de ce dernier ont été prises de manière à décrire différentes facettes de la personne ou du lieu, l'image fonctionne selon trois critères: le cadre, le plan et plan approché.

« Interpréter les images séquentielles » Ce type d'activité permet à l'étudiant d'apprendre la lecture d'une image, pour interpréter ce qu'il voit. Ainsi, l'étudiant utilise son sens de l'observation pour ensuite faire des liens et comprendre l'action.

On souhaite que l'étudiant raconte ce qui se passe plutôt que d'énumérer les personnages ou les objets de l'illustration.

Pour ce type d'activité, il est important de questionner l'étudiant. On préfère utiliser les questions « ouvertes » qui demandent de fournir une réponse plus détaillée que les questions « fermées » où l'étudiant peut répondre par oui ou non.

\section{Le cadre pratique : L'étude expérimentale}

\section{La préparation des instruments de la recherche :}

\section{(1) - Liste d'habiletés d'interprétation des images séquentielles de la langue francaise}

Le chercheur a élaboré une liste d'habiletés d'interprétation des images séquentielles de la langue française (10 items) nécessaires aux futurs enseignants, section de français. (Annexe 1)

- Pour élaborer cette liste on a consulté même un certain nombre de livres et de recherches qui traitent ces compétences. 


\section{(2) - Liste d'habiletés de la cohérence textuelle de la langue francaise}

Le chercheur a élaboré une liste d'habiletés de la cohérence textuelle (11 items) nécessaires aux étudiants de l'échantillon. (Annexe 2).

- Pour élaborer cette liste, on a consulté également un certain nombre de livres et d'études qui traitent ces compétences.

(3) -Test de la cohérence textuelle et d'interprétation des images séquentielles de la langue francaise

Pour déterminer le niveau de la cohérence textuelle et interprétation des images séquentielles , la production écrite des futurs enseignants étaient de forme narrative et les sujets étaient imposé

Afin de préparer le test de l'interprétation des images séquentielles et de la cohérence textuelle de la langue française, le chercheur a suivi le processus suivant:

Déterminer l'objectif du test, la construction du test, la durée du test et l'expérimentation du test.

Le chercheur a d'abord appliqué le test à un échantillon qui se compose de 20 étudiants puis il a réappliqué le test sur le même échantillon après trois semaines en 2020, ensuite il a calculé le coefficient de corrélation entre les notes des étudiants dans les deux passations du test et enfin, le chercheur a calculé la validité du test.

On peut constater que la corrélation est significative au niveau de 0.01 , c'est-à-dire la stabilité est élevée

L'objectif du test de la cohérence textuelle et d'interprétation des images de la langue francaise: Ce test vise à évaluer le niveau l'interprétation des images séquentielles et de la cohérence textuelle des futurs enseignants, section de français, faculté de pédagogie de Minia.

Construction du test d'interprétation des images séquentielles et de la cohérence textuelle de la langue francaise.

Le test comprend 2 parties, la première partie porte sur l'interprétation des images séquentielles., la $2^{\text {ème }}$ partie porte sur la cohérence textuelle.

\section{Pour la première partie les habiletés des étudiants interpréter les images séquentielles}

On s’intéresse ici à l'interprétation des images séquentielles.

\section{$\underline{\text { L'étudiant est invité à : }}$}

1)-Décrire différentes facettes de la personne ou du lieu, l'image fonctionne

2) -Donner une appréciation subjective de l'image. 
3)-Déterminer le sens caché ou figuré aux images qu'il perçoit.

\section{Pour La 2ème partie les habiletés des étudiants de la cohérence textuelle}

On s'intéresse ici à la cohérence textuelle dans la production écrite des futurs enseignants, section de français, faculté de pédagogie de Minia.

\section{$\underline{\text { L'étudiant est invité à : }}$}

1)-Comprendre une histoire (étapes, ordre chronologique, etc.).

2)-Organiser les idées.

3)-Utiliser des indices (faire des liens et anticiper).

4) -Organiser la tâche, placer les images (de gauche à droite).

\section{$\underline{\text { Passation du test }}$}

Le chercheur a choisi par hasard 20 futurs enseignants de la faculté de pédagogie de Minia, Ces étudiants sont inscrits en 3ème année du département de français pour l'année universitaire 2020-2021.

L'application du test comme étude pilote a permis de calculer la durée de l'application du test, sa fidélité et sa validité.

\section{$\underline{\text { Durée du test de la cohérence textuelle et de l'interprétation des images séquentielles }}$}

Pour déterminer le temps du test, le chercheur a appliqué la formule suivante :

Le temps moyen $=$ le temps mis par le premier étudiant + le temps mis par le dernier étudiant $/$ $2=40+40 / 2=40$ minutes.

Test de l'interprétation des images séquentielles $=10 \mathrm{mn}$

Test de la cohérence textuelle $\quad=30 \mathrm{mn}$

Pré test va durer environ 40 minutes.

\section{Le calcul de l'indice de fidélité et de validité :}

\section{$\underline{\text { Sources du test }}$}

Ce test est élaboré à travers les livres français, les références et les méthodes employées pour les débutants notamment les méthodes d'entrainement aux épreuves de (DELF) qui porte sur les critères de la production écrite (toujours les livres de A2 et B1)...etc. 


\section{Passation pilote du test pour le valider.}

Le chercheur a opéré la validation du test par la manière d'application et de ré-application le test au cours d'un intervalle de trois semaines sur un échantillon de futurs enseignants (étude pilote) compose de 20 étudiants, Cette passation pilote a pour objet de calculer la fidélité, la validité,

Fidélité du test : on a employé l'équation de Person comme suit :

$\mathrm{R}=$

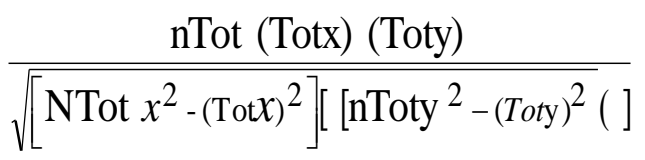

$\mathrm{R}=$ coefficient de corrélation $\quad \mathrm{N}=$ nombre des étudiants

Tot $=$ total des notes des étudiants. $\quad \mathrm{X}=$ pré - application.

$\mathrm{Y}=$ post - application

Le chercheur a calculé le coefficient de corrélation entre les deux passations en appliquant l'équation précédente de Person .

On a remarqué que la fidélité du test d'interpréter les images séquentielles et de la cohérence textuelle est .0.64.

\section{$\underline{\text { Validité du test de l'interprétation des images séquentielles et la cohérence textuelle }}$}

La fidélité du test $\quad 0.0 .64$

Le chercheur a remarqué que la fidélité est 0.0.64 ce qui signifie que le test est fidèle, Pour vérifier de la validité du test on a appliqué la formule suivante :

La validité du test $=\sqrt{\text { la fidelite }}=\sqrt{0.64}=0.8$

Cette valeur confirme que le test est valide.

\section{Notation}

Le chercheur a élaboré aussi un barème de correction de ce test, (Annexe 3-b) après avoir ramasser les réponses, il apprécie, puis met la note.

\section{$\underline{\text { Les critères de notation }}$}

Le test mesure les 3 compétences écrites concernant l'interprétation des images séquentielles et la cohérence textuelle de la langue française : 
La cohérence textuelle de la langue française : $\quad$ (10 points)

L'interprétation des images séquentielles (10 points)

-La chronologie des actions dans une consigne complexe qui ne respecte pas l'ordre des mots. etc.

(10 points)

\section{$\underline{\text { L'expérimentation du test }}$}

Le test est pratiqué 2 fois, la première fois (le pré test) était appliquée au début de l'expérimentation, il a duré 1 cours de 40 minutes, et la deuxième ré application (le post test) qui a eu lieu après 8 semaines a duré 40 minutes sur 20 étudiants.

\section{(4) -Test de la production écrite de la langue francaise (pré / post test)}

Pour déterminer le niveau actuel de la production écrite des futurs enseignants, section de français

Le test se compose d'une seule question, Cette question demande une réponse ouverte, on a accepté chaque réponse convenable et logique, mais on a pris en considération les fautes d'orthographe, de la grammaire et de la pertinence.

\section{$\underline{\text { La correction du test }}$}

Le test se compose d'une seule question.

La question (20 points), Le Total $=20$ points.

Le chercheur a utilisé une grille de correction extraite des épreuves de (DELF) qui porte sur les critères de la production écrite (Annexe 4-b)

La grille de correction comprend 10 critères et un score total

Les critères de la correction suivent chacun une échelle descriptive graduée en 5 catégories (scores de 0 à 2)

\section{Durée du test de La production écrite de la langue francaise :}

\section{Le calcul du temps :}

Pour déterminer le temps du test, nous avons applique la formule suivante :

Le temps moyen = le temps mis par le premier étudiant + le temps mis par le dernier étudiant $=90 / 2=45$ minutes.

\section{$\underline{\text { Validité du test }}$}

Le chercheur a d'abord appliqué le test a un échantillon composé de 20 étudiants puis il a ré appliqué le test sur le même échantillon après huit semaines en 2021 ensuite il a calculé le 
coefficient de corrélation entre les notes des étudiants dans les deux passations du test et enfin il a calculé la validité de correction du test

La stabilité du test $=0.97$

La validité total du test $=\sqrt{\text { la fidelite }}=\sqrt{0.97}=0.98$

On peut constater que la corrélation est significative au niveau de 0.01 , c'est-à-dire la stabilité est élevée.

\section{(5) - Le programme expérimental}

\section{Présentation générale}

Ces cours s'adressent aux futurs enseignants, section de français à la faculté de pédagogie de Minia. Ces étudiantes ont déjà étudié le français au cycle secondaire comme deuxième langue étrangère et aussi pendant 2 ans au cours de leur étude à la faculté de pédagogie.

\section{Les objectifs généraux}

\section{On peut déterminer les objectifs généraux dans les points suivants :}

1) Développer l'interprétation des images séquentielles et la cohérence textuelle.

2) Améliorer la qualité du français écrit par un travail sur la cohérence du texte.

3) Reconnaître l'effet d'un programme proposé sur la production écrite chez les futurs enseignants section du français, faculté de pédagogie de Minia.

\section{Objectifs Opérationnels}

\section{L'étudiant ayant étudié ce programme d'entrainement est censé être capable de:}

1) Présenter un texte en le divisant en paragraphes.

2) Le respect de la structure du texte (introduction-développement-conclusion)

3) Le texte présente les éléments de la situation initiale (temps, lieu, personnage), un processus de transformation, la situation finale.

4) Cohérence et cohésion relier une série d'éléments courts, simples et distincts en un discours qui s'enchaîne.

5) Progresser un texte (des éléments pertinente, l'enchainement des idées, la non contradiction etc.)

6) La bonne utilisation des connecteurs.

7) Maîtriser l'emploi des temps. 
8) Grammaire et syntaxe

Accord en genre et en nombre, pronoms, marques verbales, etc.

9) Le ménagement des transitions

10) Mettre en considération l'orthographe lexicale, la ponctuation et la mise en page (la majuscule, l'apostrophe le trait d'union, l'utilisation adéquate des abréviations etc.)

\section{$\underline{\text { 1. Contenu du cours }}$}

- Ce programme vise à améliorer des habiletés de l'interprétation des images séquentielles et la cohérence textuelle.

- Le cours expérimental de français écrit a été un cours de 45 heures, dispensé de façon collective à raison de trois heures par semaine. Il était basé sur la perspective globale du texte et sur les principes de la cohérence textuelle.

-Les éléments qui composent la cohérence textuelle font l'objet de modules d'apprentissage.

-On commence par une évaluation du niveau de l'interprétation des images séquentielles et la cohérence textuelle.

-Les premiers cours sont consacrés à des exercices de l'interprétation des images séquentielles et la cohérence textuelle

-Une série de tests et d'exercices, pour renforcer le système de la cohérence textuelle, Les obstacles à la lecture des images séquentielles.

-Des exercices pour développer la cohérence textuelle,

- les futurs enseignants section du français, faculté de pédagogie de Minia reçoivent une rétroaction immédiate.

-Sur leurs résultats pour développer l'interprétation des images séquentielles, et la cohérence textuelle, les étudiants sont invités à raconter l'histoire de ces images séquentielles mieux qu'au départ.

-Les cours consisteront en exercices pratiques collectifs et individuels. Des images séquentielles choisies pour leur intérêt thématique, leur apport terminologique leurs caractéristiques seront analysées en classe.

-Chaque étudiant doit être habitué à parler et comprendre en français afin de découvrir le sens des notions que l'on utilise. 


\section{Contenu spécifique}

Ce programme proposé comporte 3 modules|: ces modules sont formulés pour développer quelques habiletés de la cohérence textuelle et l'interprétation des images séquentielles en classe de langue. Ces modules sont intitulés.

\section{$\underline{\text { Module } 1}$}

Dans ce module le chercheur a mis l'accent sur Le travail au plan de la pensé concernant la Cohérence du texte à travers les habiletés de la cohérence textuelle et l'interprétation des images séquentielles « clarifier la compréhension du sujet à traiter, déterminer le thème, rassembler les idées sur le sujet et écrire le texte. ».

\section{$\underline{\text { Module } 2}$}

$\underline{\text { Dans ce module le chercheur a mis l'accent sur }}$

La vérification des points particuliers de sa production tels les liens entre les idées, la progression du sujet, la structure des phrases, les accords, etc. c ._à .d évaluer les points forts et les points faibles de sa production

-Interpréter les images séquentielles

-remédier les fautes de cohérence textuelle les plus habituelles dans les copies des futurs enseignants de la faculté de pédagogie de Minia.

\section{$\underline{\text { Module } 3}$}

$\underline{\text { Dans ce module le chercheur a mis l'accent sur l'entraînement à: }}$

-Maîtriser l'emploi des temps en réalisant l'interprétation et la cohérence textuelle

- La bonne utilisation des connecteurs.

- récriture du texte.

\section{Critères de sélection}

Le chercheur a utilisé une méthode simple basée sur des principes qui ont fait leurs preuves en pédagogie.

- Des activités linguistiques nombreuses et intéressantes qui favorisent la lecture d'images séquentielles ;

- Des tests formatifs avec des rétroactions immédiates sur le progrès dans la cohérence textuelle et l'interprétation des images fixes. 
- Les images séquentielles sont généralement d'une longueur moyenne, c.à.d. qu'ils se composent de quatre images séquentielles ou moins. Souvent, ils sont suivis d'exercices qui permettent d'acquérir un vocabulaire d'analyse .

D'une part, les images ne sont pas trop, D'autre part, les thèmes abordés renvoient au vécu des apprenants puisque les documents traitent tous les sujets susceptibles d'intéresser des jeunes.

\section{Le déroulement du programme}

Le chercheur a appliqué le programme d'entrainement expérimental proposé selon un emploi de temps précis, le programme se compose de 3 modules chaque module contient quelques habiletés de la lecture d'images séquentielles., le chercheur a noté devant chaque module le temps nécessaire pour l'appliquer.

\section{$\underline{\text { L'enseignement du programme comprend les étapes suivantes }}$}

1) Le chercheur demande aux étudiants de regarder bien les images séquentielles qui ne sont pas accompagnées d'un texte puis, ils vont répondre à ce qui suit :

Qu'est-ce que vous voyez? Relève le titre de ces images. De quoi parle ces images?

2) A travers ce premier contact avec les images séquentielles, le chercheur amène les étudiants à formuler des hypothèses de sens.

3) Une lecture orale exemplaire sera faite par le chercheur suivie par une lecture individuelle entrecoupée par des questions de compréhension et par des corrections systématiques afin de vérifier les hypothèses de sens.

4) Le chercheur passe ensuite à l'explication progressive du texte par un jeu de questionsréponses.

5) L'analyse du texte proposé doit se terminer par une courte synthèse que les étudiants porteront sur leurs cahiers, cette synthèse peut revêtir plusieurs formes, le plus souvent des exercices lacunaires.

6)L'application d'un programme d'entraînement proposé à la lecture des images séquentielles pour améliorer la cohérence textuelle de la langue française.

7) Compter les taux la cohérence textuelle réelle dans la production écrite des étudiants de l'échantillon, les taux de l'interprétation des images séquentielles.

8)) Traitement statistique des résultats.

9) Interpréter les résultats. 


\section{Evaluation : L'évaluation de ce programme}

On utilise l'évaluation afin de vérifier l'acquisition des habiletés et l'interprétation des images séquentielles et de la cohérence textuelle chez les apprenants.

\section{Dans ce programme on a trois types d'évaluation :}

1) A travers le pré- test en vue de déterminer le niveau initial des individus de l'échantillon dans les habiletés de la cohérence textuelle et d'interprétation,

Le chercheur a pu évaluer la cohérence d'un texte produit par le futur enseignant.

2) Le chercheur a utilisé l'évaluation formative qui vise à aider les étudiants réutiliser l'acquis dans un contexte nouveau.

On fait cette évaluation pendant l'exécution du programme et à la fin de chaque séance !

3) Le chercheur a utilisé l'évaluation sommative à la fin du programme en appliquant le posttest.

A travers cette évaluation le futur enseignant devenir plus conscient des éléments qui assurent la cohérence des textes qu'il lit.

Cette recherche s'est concentrée sur quelques activités langagières à partir les images séquentielles en ce qui concerne le développement de la cohérence textuelle et d'interprétation.

\section{$\underline{\text { L'application du test. }}$}

Après l'application du programme, le chercheur a appliqué le post test sur le même échantillon

\section{Le Guide pédagogique (Annexe no: 6)}

Le chercheur a élaboré un guide pédagogique à ce programme qui explique les démarches suivies dans ce programme, et comment développer l'interprétation et la cohérence textuelle.

$\underline{\text { Ce guide comporte le suivant : }}$

- Les objectifs du programme, le contenu du programme, les exercices proposés et l'évaluation

Ce guide se compose de deux parties : le cahier d'étudiant et le livre d'enseignant

Le cahier d'étudiant comporte :

- Les objectifs de chaque module, le contenu lexical de ce module, le contenu grammatical, le contenu de programme (les 3 modules) et un certain nombre d'exercices sur chaque module.

$\underline{\text { Tableau de description temporelle de l'expérimentation }}$ 


\begin{tabular}{|c|c|c|}
\hline & \multicolumn{2}{|c|}{ Le temps } \\
\hline & Par minutes & Par cours (environ) \\
\hline $\begin{array}{l}\text { Module 1: } \\
\text { Entraînement à } \\
\text {-Présenter un texte en le divisant en } \\
\text { paragraphes, } \\
\text {-Le respect de la structure du texte } \\
\text { (introduction-développement- } \\
\text { conclusion), } \\
\text {-Le texte présente les éléments de } \\
\text { la situation initiale (temps, lieu, } \\
\text { personnage), } \\
\text { un processus de transformation, la } \\
\text { situation finale, } \\
\text {-Le texte progresse (des éléments } \\
\text { pertinente, l'enchainement des } \\
\text { idées, le non contradiction, } \\
\text { Ménagement des transitions. }\end{array}$ & 180 minutes & $\begin{array}{l}\text { Ce module comprend } \\
2 \text { cours }\end{array}$ \\
\hline $\begin{array}{l}\text { Module 2: } \\
\text { Entraînement à } \\
\text {-Interpréter les images séquentielles } \\
\text {-remédier les fautes de cohérence } \\
\text { textuelle les plus habituelles dans } \\
\text { les copies des étudiants de la } \\
\text { faculté de pédagogie de Minia }\end{array}$ & 180 minutes & $\begin{array}{l}\text { Ce module comprend } \\
2 \text { cours }\end{array}$ \\
\hline $\begin{array}{l}\text { Module 3: } \\
\text { Entraînement à: } \\
\text {-Maîtriser l'emploi des temps en } \\
\text { réalisant la cohérence textuelle } \\
\text {-La chronologie des actions dans } \\
\text { une consigne complexe qui ne } \\
\text { respecte pas l'ordre des mots. etc. }\end{array}$ & 180 minutes & $\begin{array}{l}\text { Ce module comprend } \\
2 \text { cours }\end{array}$ \\
\hline L'évaluation du programme & & \\
\hline $\begin{array}{l}\text { Test de l'interprétation des images } \\
\text { séquentielles et de la cohérence } \\
\text { textuelle. } \\
\text { Pré-test } \\
\text { Post -test }\end{array}$ & $\begin{array}{l}40 \text { minutes } \\
40 \text { minutes }\end{array}$ & $\begin{array}{l}1 \text { cours } \\
1 \text { cours }\end{array}$ \\
\hline $\begin{array}{l}\text { Test de la production écrite } \\
\text { Pré-test } \\
\text { Post- test }\end{array}$ & $\begin{array}{l}45 \text { minutes } \\
45 \text { minutes }\end{array}$ & $\begin{array}{l}1 \text { cours } \\
1 \text { cours }\end{array}$ \\
\hline Total & 710 minutes $=11$ & 10 cours \\
\hline
\end{tabular}




\section{$\underline{\text { Analyse et interprétation les résultats }}$}

\section{Pour répondre aux questions de la recherche}

\section{1) 1) Pour répondre à la $1^{\text {ère }}$ question,}

Quels sont les critères sur lesquels le chercheur s'appuie pour juger l'interprétation des images séquentielles chez les futurs enseignants de la faculté de pédagogie de Minia section de français?

Le chercheur a déterminé les habiletés de l'interprétation des images séquentielles nécessaires aux les futurs enseignants de la faculté de pédagogie; et ceci à travers la lecture des recherches et des études antérieures qui ont traité ce sujet. (Annexe No : 1)

\section{$\underline{\text { La version finale de ces habiletés comporte : }}$}

1- le décodage des images séquentielles.

2-Les capacités de représentation mentale.

3-Le raisonnement logique.

4-Trouver un titre aux images séquentielles.

5- Décrire Tout ce qui est perçu par les étudiants (les objets, les êtres, les proportions, les couleurs, etc. ;) Ce qui est connoté par étudiants.

6-Décrire les émotions que les images séquentielles peuvent susciter.

7- Décrire les interprétations possibles des images séquentielles.

8-Rédiger sur un brouillon ce que l'étudiant a compris des images séquentielles.

9-Décrire les interprétations possibles des images séquentielles.

2) Pour répondre à la $2^{\text {ème }}$ question,

Quels sont les critères sur lesquels le chercheur s'appuie pour juger la cohérence d'un texte de futurs enseignants?

Le chercheur a déterminé les habiletés de la cohérence textuelle de la langue française nécessaires aux futurs enseignants de la faculté de pédagogie de Minia ; et ceci à travers la lecture des recherches et des études antérieures qui ont traité la cohérence textuelle de la langue française. (Annexe No:2)

\section{La version finale à ces compétences comporte :}

\section{L'étudiant ayant étudié ce programme est censé être capable de:}

1) Présenter un texte en le divisant en paragraphes

2) Le respect de la structure du texte (introduction-développement-conclusion) 
3)Le texte présente les éléments de la situation initiale (temps, lieu, personnage), un processus de transformation, la situation finale

4) Cohérence et cohésion relier une série d'éléments courts, simples et distincts en un discours qui s'enchaîne

5) Progresser un texte (des éléments pertinents, l'enchainement des idées, la non contradiction etc.)

6) La formulation claire des informations

7) Maîtriser de l'emploi des temps

8) Grammaire et syntaxe

Accord en genre et en nombre, pronoms, marques verbales, etc.

9) Le ménagement des transitions

10) Mise en considération de l'orthographe lexicale, la ponctuation et la mise en page (la majuscule, l'apostrophe le trait d'union, l'utilisation adéquate des abréviations etc.)

\section{3) Pour bien répondre à la $3^{\text {ème }}$ question}

Quel est le degré de maîtrise des habiletés de l'interprétation des images séquentielles et la cohérence textuelle que possède l'apprenant

Le chercheur a appliqué une étude-pilote au cours de l'année universitaire 2020/2021 aux futurs enseignants de la faculté de pédagogie à l'université de Minia. (20 étudiants). Cette étude-pilote se compose d'un test de l'interprétation et la cohérence textuelle de la langue française, ayant pour but d'enquêter le niveau de la cohérence textuelle et l'interprétation des images séquentielles réelle chez ces étudiants.

Cette étude a confirmé le manque des habiletés de l'interprétation de la langue française, et aussi de la cohérence textuelle chez les étudiants de $3^{\text {ème }}$ année de faculté de pédagogie à l'université de Minia. Ce manque d'habiletés peut revenir de l'utilisation des stratégies d'enseignement inconvenables à leurs styles d'apprentissage.

\section{4) Pour répondre à la $4^{\text {ème }}$ ème question}

Quel est l'effet de l'utilisation d'un programme proposé à la lueur des images séquentielles pour développer l'interprétation des images séquentielles et la cohérence textuelle chez les futurs enseignants faculté de pédagogie section de français ?

Le programme d'entraînement proposé a un effet positif pour améliorer la cohérence textuelle et l'interprétation des images séquentielles de la langue française.

Le coefficient de corrélation entre les résultats de la cohérence textuelle après l'application des modules proposés, et le post et l'interprétation des images séquentielles de la langue française est - 0. 504. 
La valeur de l'influence est 0.97 au test de la cohérence textuelle et 0.97 au test de la compréhension écrite, ce sont des taux élevés. La significativité est au niveau de 0.01 .

\section{5) Pour répondre à la $5^{\text {ème }}$ question}

Quel est l'effet de l'utilisation de ce programme proposé sur la production écrite chez. les futurs enseignants section de français, faculté de pédagogie de Minia?

Le chercheur a appliqué un pré/post test de la production écrite pour déterminer le niveau la production écrite de la langue française chez les futurs enseignants section de français faculté de pédagogie de Minia avant et après l'application du programme suggéré .

Les habiletés de la production écrite de la langue française mettent l'accent sur:

\section{1) Le contenu}

Le contenu du texte basé sur le choix et la pertinence des idées à transmettre

\section{2) Le style}

Le style, c'est l'utilisation de plusieurs éléments linguistiques (ex. : synonymes, divers types de phrases) pour donner au texte une touche originale et personnelle afin de susciter et de maintenir l'intérêt du public cible.

\section{3) L'organisation du texte}

Un agencement et un enchaînement logiques et pertinents des idées ou des informations. Les liens entre ces idées devraient préciser davantage son message.

\section{4) Les règles de langue}

L'emploi correct de l'orthographe d'usage et des règles grammaticales, la ponctuation, le lexique et la syntaxe afin que le message à transmettre soit compris sans ambiguïté.

Le coefficient de corrélation entre les résultats du test des habiletés de l'interprétation, et de la cohérence textuelle de la langue française après l'application du programme proposé, et le post test de la production écrite est significatif. La valeur de l'influence au test d'habiletés de l'interprétation et de la cohérence textuelle de la langue française et au test de la production écrite a des taux très élevés. La significativité est au niveau de 0.001 , Ce qui met en valeur l'efficacité du programme proposé et son influence à développer les habiletés de l'interprétation et de la cohérence textuelle de la langue française et à améliorer la production écrite chez les étudiants de l'échantillon. 


\section{Résultats du pré/post test}

\section{L'analyse porte sur:}

\section{L'analyse porte sur}

- Les progrès réalisés entre le pré- test et le post- test

- L'effet de l'utilisation d'un programme proposé pour développer quelques habiletés de l'interprétation de la langue française et la cohérence textuelle à la lueur des images séquentielles et son effet sur la production écrite chez l'échantillon

Le chercheur a analysé les résultats obtenus après la passation du pré/post test de l'interprétation et de la cohérence textuelle de la langue française.

Pour l'analyse statistique des résultats, le chercheur a utilisé le programme statistique SPSS pour calculer la moyenne arithmétique des notes, l'écart type et la valeur de T.

\section{Le chercheur a présenté et analysé la première hypothèse suivante :}

1- Il existe une différence significative au seuil de $(0,01)$ entre la moyenne des notes des futurs enseignants de la faculté de pédagogie de Minia au (pré-post) test des habiletés de l'interprétation de la langue française, en faveur du post-test.

Tableau (1)

Les résultats relatifs au pré /post de Test de l'interprétation

\begin{tabular}{|r|r|r|r|r|r|r|r|r|r|}
\hline & $\mathrm{N}$ & Min & Max & Ma & E & TN & DL & T & S \\
\cline { 1 - 8 } Pré -test & 20 & 6 & 10 & 8.40 & 1.27 & 20 & 19 & 14.32 & $\begin{array}{c}\text { Au niveau } \\
\text { de } 0.001\end{array}$ \\
\cline { 1 - 7 } Post-test & 9 & 13 & 11.50 & 1.39 & & & & \\
\cline { 3 - 7 } & & & & & & & & & \\
\hline
\end{tabular}

$\mathrm{N}=$ nombre des étudiants.

Max=maximum.

$\mathrm{E}=$ écart type.

$\mathrm{Dl}=$ degré de liberté.

$\mathrm{S}=$ significative.
Min =minimum

$\mathrm{Ma}=$ moyenne arithmétique.

Tn $=$ total des notes.

$\mathrm{T}=$ valeur de $(\mathrm{t})$ test.

Ns = non significative.

- Les progrès réalisés entre le pré- test et le post- test.

Le chercheur a remarqué que les étudiants souffrent de l'insuffisance de leur habileté interprétative, il semble que l'habitude de la lecture des images séquentielles en langue arabe chez les étudiants n'est pas automatiquement transférable à la lecture de images séquentielles en français. 
Alors la formation en français que l'apprenant égyptien de français a suivi ne l'aide pas à faire une lecture critique des images séquentielles en français, il est dépourvu d'esprit de synthèse et de réflexion critique, son manque de réflexion personnelle devant un texte rend difficile la pédagogie de la lecture des images séquentielles en français.

Malgré tout ça, il y a une bonne progression aux quelques tâches de l'interprétation de la langue française des images séquentielles (le décodage des images séquentielles, le raisonnement logique, Les capacités de représentation mentale ...etc.).

On peut remarquer une progression dans quelques habiletés de l'interprétation permettent aux apprenants de FLE de s'exprimer aisément.

Le chercheur a trouvé des Interprétations multiples différemment par chaque lecteur des images séquentielles selon sa personnalité, de sa sensibilité ou l'indécidable (le retour au texte permet plusieurs interprétations toutes acceptables). Pour faire face à ce phénomène, le chercheur a essayé de mettre en discussion et à essayer d'argumenter son interprétation.

Les résultats obtenus montrent que la performance aux tâches de l'interprétation de la langue française des images séquentielles est significativement supérieure à celle des tâches de la cohérence textuelle chez les étudiants de la $3^{\text {ème }}$ année de faculté de pédagogie à l'université de Minia.

\section{Commentaire du tableau :}

Les résultats précédents montrent le minimum et le maximum des notes obtenues par l'échantillon de la recherche dans les deux tests (pré/post tests), la moyenne arithmétique, l'écart type, le total des notes de chaque test, les degrés de liberté, et enfin la valeur de $\mathrm{T}$ au niveau 0.001 .

La valeur de $(\mathrm{T})$ au niveau de 00.1 est significative. C'est un taux très élevé.

Ce qui met en valeur la progression de l'habileté interprétative des étudiants due à l'appréciation du programme proposé et son influence sur le niveau de la production écrite des étudiants.

\section{Par suite, la première hypothèse de la recherche a été vérifiée.}

\section{Le chercheur a présenté et analysé aussi la deuxième hypothèse suivante:}

2- Il existe une différence significative au seuil de $(0,01)$ entre la moyenne des notes des futurs enseignants de la faculté de pédagogie de Minia au (pré-post) test d'habiletés de la cohérence textuelle de la langue française, en faveur du post-test. 
Tableau (2)

Les résultats relatifs au pré /post de Test de la cohérence textuelle

\begin{tabular}{|c|c|c|c|c|c|c|c|c|c|}
\hline & $\mathrm{N}$ & Min & Max & Ma & $\mathrm{E}$ & $\mathrm{TN}$ & DL & $\mathrm{T}$ & S \\
\hline Pré -test & \multirow{2}{*}{$\begin{array}{l}2 \\
0\end{array}$} & 5 & 9 & 6.30 & 1.32 & \multirow[t]{2}{*}{20} & \multirow[t]{2}{*}{19} & \multirow[t]{2}{*}{12.42} & \multirow{2}{*}{$\begin{array}{c}\mathrm{Au} \\
\text { niveau } \\
\text { de } 0.001\end{array}$} \\
\hline Post-test & & 9 & 11 & 10 & .725 & & & & \\
\hline
\end{tabular}

Le chercheur a analysé les résultats obtenus avant et après la passation du test de la cohérence textuelle de la langue française, des futurs enseignants de la faculté de pédagogie de Minia, département de français.

On peut remarquer une progression dans la cohérence textuelle de la langue française, Il y a une amélioration significative remarquable des habiletés de la cohérence textuelle de la langue française (par comparaison avec les résultats des habiletés de de la cohérence textuelle de la langue française initiale)

Le chercheur affirme que la cohérence textuelle est la relation harmonieuse et une suite logique existantes entre les phrases et les différentes parties d'un texte, faisant de lui un tout. Dans un texte cohérent, il ne peut exister des contradictions, ni désintégration entre les différentes parties. Pour qu'un texte remplisse les conditions de la cohérence textuelle, il faut qu'il remplisse quatre règles suivantes : avoir une progression de l'information, avoir une relation étroite entre les passages et les idées, avoir un champ lexical et la non-contradiction.

On trouve que l'entraînement proposé a permis aux groupes entraînés, d'améliorer significativement leurs performances de l'interprétation des images séquentielles et de la cohérence textuelle, et également dans la production écrite.

\section{Le chercheur a présenté et analysé aussi la troisième hypothèse suivante:}

3- Il existe une différence significative au seuil de $(0,01)$ entre la moyenne des notes des futurs enseignants de la faculté de pédagogie à la production écrite avant et après l'enseignement du programme en faveur du post test.

\section{Tableau (3)}

Les résultats relatifs au pré /post test de la production écrite

\begin{tabular}{|c|c|c|c|c|c|c|c|c|c|}
\hline & $\mathbf{N}$ & Min & Max & Ma & $\mathbf{E}$ & TN & DL & $\mathbf{T}$ & $\mathbf{S}$ \\
\hline Pré -test & \multirow[t]{2}{*}{20} & 9 & 13 & 10.9 & 1.33 & \multirow[t]{2}{*}{20} & \multirow[t]{2}{*}{19} & \multirow[t]{2}{*}{25.66} & \multirow{2}{*}{$\begin{array}{c}\text { Au } \\
\text { niveau } \\
\text { de } 0.001\end{array}$} \\
\hline Post-test & & 13 & 16 & 14.75 & .967 & & & & \\
\hline
\end{tabular}


Dans une recherche didactique comme la nôtre, le chercheur a analysé les résultats obtenus avant et après la passation du test de la production écrite de la langue française des futurs enseignants de la faculté de pédagogie de Minia, département de français.

$\mathrm{Au}$ début de l'expérimentation le chercheur a trouvé que la plupart des futurs enseignants rencontrent des obstacles lors de leurs productions écrites, sur le plan de l'organisation textuelle, la progression thématique, et la continuité logique des informations.

Apres avoir appliqué le programme proposé, on peut remarquer une progression dans la production écrite de la langue française,

Il y a une amélioration significative remarquable dans les habiletés de la production écrite (par comparaison avec les résultats des habiletés de la production écrite initiale).

On trouve que le programme proposé a permis aux groupes entraînés,

de produire un texte avec un raisonnement logique et une cohérence textuelle.

On peut affirmer qu'un texte devient incohérent lorsqu'il est difficile de trouver une relation entre des informations fournies

\section{A travers ce tableau on peut dire que :}

Les résultats précédents montrent aussi le minimum et le maximum des notes obtenues par de l'échantillon de la recherche dans les deux tests (pré/post tests), la moyenne arithmétique, l'écart type, le total des notes de chaque test, les degrés de liberté, la valeur de T et enfin la significativité au niveau 0.001

La valeur de $(\mathrm{T})$ au niveau de 00.1 est significative.

Ce qui met en valeur la progression de l'interprétation de la langue française des images séquentielles due à l'appréciation du programme proposé et son influence sur le niveau de la cohérence textuelle des étudiants.

Après l'analyse des réponses des futurs enseignants aux tests de l'interprétation de la langue française des images séquentielles de la langue française (post application) et la cohérence textuelle (post application), on peut dégager ce qui suit

En ce qui concerne le premier axe (l'interprétation de la langue française des images $\underline{\text { séquentielles) }}$

En appliquant le programme proposé des images séquentielles ayant pour but de développer les habiletés de l'interprétation des images séquentielles des futurs enseignants de la faculté de pédagogie de Minia, On peut remarquer que:

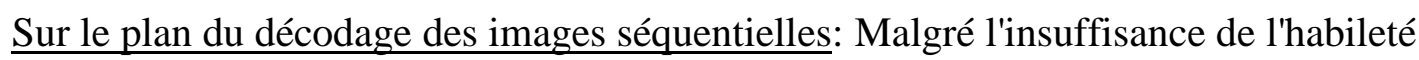
interprétative chez les étudiants de l'échantillon, le chercheur a observé des progrès marqués 
chez un certain nombre des futurs enseignants grâce à l'entrainement continu des activités des images séquentielles.

Sur le plan des capacités de représentation mentale: Un grand nombre d'étudiants s'intéresse au côté pratique de la langue à travers les images séquentielles, ils ont pu employer leurs capacités de représentation mentale à raconter des histoires, à imaginer une fin d'une histoire d'une manière logique.

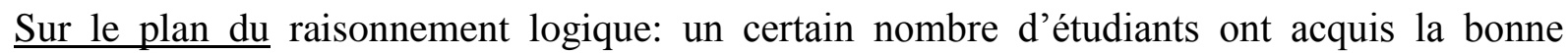
chronologie au rappel des événements et sérialisation réussie, les inférences mobilisant des capacités de synthèse et demandant de faire des liens entre des informations éloignées les unes des autres.

Sur le plan de «Trouver un titre aux images séquentielles »: on affirme qu'il n'y a pas de grands obstacles dans cette habileté. Puisque les étudiants de l'échantillon ont bien maitrisé le code de comprendre, ils ont étudié la langue française depuis 2 ans. Donc, ils ont un bagage lexical, on l'a utilisé, pour trouver des bon titres aux images séquentielles en racontant les histoires dans le programme.

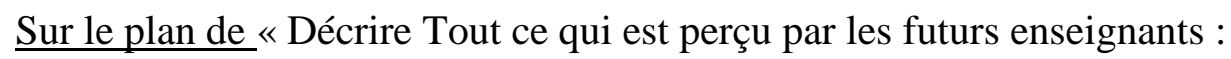

La plupart d'étudiants (qui savent trier les informations importantes et donnent plus de détails) n'ont pas d'obstacles dans cette habileté aussi puisqu'ils maitrisent bien la langue qui leur aide à la description (leur niveau actuel est presque A2+).

Sur le plan de «Décrire les émotions que les images séquentielles peuvent susciter» l'émotion est un trouble, une agitation de l'esprit et du corps en réaction à un événement une idée; le sentiment est ce que l'on éprouve ce que l' on ressent face aux situations de la vie et face à autrui, quand on évoque ce que l' on ressent en racontant, on produit des descriptions et des récits subjectifs quand on ne fait aucune allusion aux émotions éprouves, on produit des descriptions et des récits neutres, La plupart des étudiants de l'échantillon ont pu exprimer les sensations les sentiments que les images séquentielles peuvent susciter d'une langue française compréhensible et acceptable.

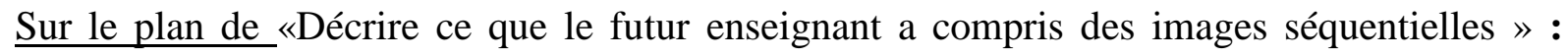
Certains étudiants lecteurs lisent et interprètent avec une rétention (des images séquentielles sans difficulté).

Sur le plan de Décrire les interprétations possibles des images séquentielles, montre également une progression attendue étant donné que (les interprétations possibles des images séquentielles) continue à évolue.

En terme de conclusion, le chercheur confirme que L'interprétation des images séquentielles a mis l'accent non seulement sur le savoir mais aussi sur le savoir-faire. 
En ce qui concerne le deuxième axe (de la cohérence textuelle):

Après avoir appliqué le programme proposé, En appliquant le post test de la cohérence textuelle, On peut remarquer que: la production écrite des futurs enseignants manque de cohérence malgré l'existence de phrases correctes et respect des normes grammaticales.

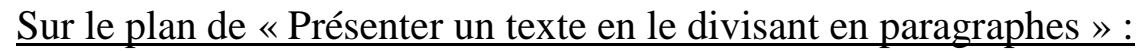

Après avoir étudié le programme proposé, la production écrite des futurs enseignants a amélioré, on trouve qu'il y a une progression remarquable a la forme de la production écrite, les étudiants ont bien divisé le texte écrit aux paragraphes.

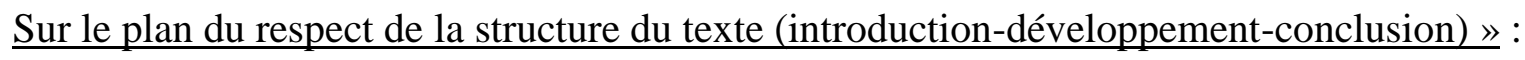

Au début de l'expérimentation la majorité des étudiants de l'échantillon à l'université sont incapables de : -

- produire une introduction cohérente du texte ;

- produire un développement cohérent du texte ;

- produire une conclusion cohérent du texte ;

- Veiller à la cohésion des parties de la phrase ;

- Enchaîner les idées.

Après avoir appliqué le programme proposé, et avec l'entrainement continue tout au long d'application du programme, on trouve qu'il y a une progression remarquable a la forme du respect de la structure du texte (introduction-développement-conclusion, les étudiants ont bien fait une bonne introduction ils ont développé le sujet propose, ils ont pu faire une conclusion cohérente et convenable au thème propose.

$\underline{\text { Sur le plan de : "Cohérence et cohésion relier une série d'éléments courts, simples et }}$ distincts en un discours qui s'enchaîne $»$ : le chercheur a trouvé que ce problème dépasse l'aspect orthographique et grammaticale, la maitrise de la langue pour eux est trop relié à apprendre des règles grammaticales il y a une difficulté à développer une idée et à élaborer un texte ordonne et logique .

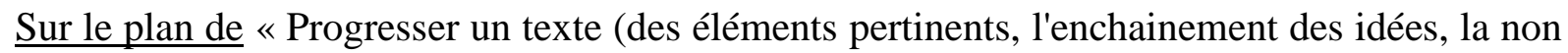
contradiction etc.) »

La production écrite des étudiants de l'échantillon manque de continuité et de progression de l'information. La plupart d'eux ne maitrisent pas les rapports logiques.

Sur le plan de «Maîtriser l'emploi des temps »: La conjugaison est une dimension qui demande un travail approfondi avec la classe, la majorité des futurs enseignants étaient 
incapables d'accorder le verbe avec son sujet ou d'identifier les principaux temps de l'indicatif. Mais à travers la correction continue de leurs fautes, la performance a amélioré.

\section{Grammaire et syntaxe}

Accord en genre et en nombre, pronoms, marques verbales, la majorité des étudiants avaient de très grandes obstacles avec la syntaxe. Certains pouvaient écrire dix lignes sans la ponctuation : ni majuscule au début des phrases, ni point à la fin, d'autres n'utilisaient la ponctuation mais de façon totalement incohérente, L'orthographe grammaticale est également un problème constant chez les étudiants.

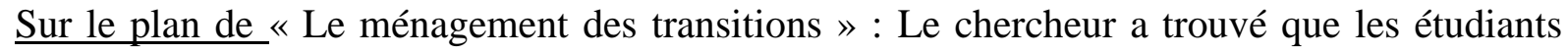
avaient de grande difficulté au management des transitions, il y a une maladresse dans le changement de thème, certains d'étudiants n'utilisent pas des phrases de transitions en changeant le thème.

Sur le plan de « Mettre en considération l'orthographe lexicale, la ponctuation et la mise en page (la majuscule, l'apostrophe le trait d'union, l'utilisation adéquate des abréviations etc.) : on note que l'habitude de l'écriture est très peu exercée, certains étudiants manifeste une difficulté particulière au plan du vocabulaire, d'autres ont des difficultés en syntaxe .A partir de la production écrite les étudiants ont pu traduire leurs pensées, la bonne réflexion aide à diminuer peu à peu les erreurs à travers la relecture et l' autoévaluation ,le chercheur a observé des progrès marqués à ce cote .

\section{En ce qui concerne le troisième axe (la production écrite) :}

Tout d'abord, la recherche actuelle a essayé de répondre à la question principale suivante :

«(Comment développer quelques habiletés de l'interprétation et la cohérence textuelle de la langue française chez les futurs enseignants de la faculté de pédagogie de Minia, section de français à travers l'utilisation des images séquentielles? Et quel est l'effet de ce programme sur la production écrite des étudiants de l'échantillon?)».

Les résultats obtenus et analysés ont confirmé clairement que l'utilisation des images séquentielles est efficace dans le domaine de développer quelques habiletés de l'interprétation et la cohérence textuelle de la langue française à travers un programme élaboré à ce propos.

On doit accorder une place importante à la production écrite des futurs enseignants de la faculté de pédagogie de Minia au niveau de la phrase et également au niveau de la textualité du texte.

La présente étude traite un problème majeur : comment améliorer les productions écrites des futurs enseignants de la faculté de pédagogie de Minia?

La réflexion porte sur trois ordres de difficultés : la structure, le contenu et l'expression. 
Le chercheur a proposé d'abord d'amener les futurs enseignants à respecter une forme cadre, de développer ensuite leur imagination et de les conduire parallèlement à progresser en expression.

À la lecture de leurs premiers travaux de la production écrite, le problème était (manque des habiletés de la production écrite et particulièrement la cohérence textuelles ...) que la tâche pour y remédier semblait insurmontable. Les problèmes étaient si multiples que le chercheur ne savait par où commencer.

Le chercheur a déterminé les difficultés des futurs enseignants, on les a analysées, on a tenté d'en chercher les causes pour ensuite proposer d'y remédier. Et enfin on a mené une réflexion axée sur la pratique

Le chercheur a travaillé progressivement sur des difficultés de trois ordres: la structure (de la forme), le contenu (du fond) et l'expression. La priorité était de donner aux futurs enseignants de la faculté de pédagogie de Minia des bases de méthode pour réaliser leurs travaux d'écriture, de leur apprendre à respecter une forme cadre dans laquelle pourrait ensuite s'épanouir leur imagination, ce qui les amènerait enfin à un travail approfondi sur leur expression.

Le chercheur a évalué chaque critère donné dans le barème qu'il a utilisé (Annexe 3) après l'analyse des productions des étudiants.

En termes de conclusion on peut affirmer que la majorité des enseignants notent des remarques grammaticales délimités et précisés, mais ils passent au niveau du texte, ils font des remarques floues, pour apporter l'aide à ces étudiants à maitriser la rédaction cohérente, il faut que l'enseignant donne des remarques précisés et détaillées concernant la cohérence textuelle en respectant la progression de l'information, la relation entre les paragraphes et les idées le champ lexical du sujet traité, et la non contradiction.

On peut affirmer également que la cohésion oblige le texte à respecter une structure, la cohérence se mesure par les interactions intervenant entre le texte et le lecteur.

Le but final est de rendre les futurs enseignant maîtriser suffisamment l'écriture pour traduire leur pensée au plus juste et qu'ils soient capables de la développer.

\section{$\underline{\text { Les recommandations de la recherche }}$}

D’après les résultats obtenus, le chercheur peut suggérer les recommandations suivantes :

1) la nécessite de développer la cohérence textuelle de la langue française à l'enseignement secondaire qui est un pont vers l'université. Si l'écrit d'un étudiant manque de textualité, c'est que l'étudiant au secondaire est mal formé pour bien écrire. 
2) élaborer un programme assisté par l'ordinateur pour développer quelques habiletés de l'interprétation de la langue française à partir des images séquentielles au cycle universitaire.

6) développer la cohérence textuelle de la langue française à travers les activités concernant les rapports logique, la progression thématique qui sont des principes fondamentaux d'un texte cohérent.

\section{$\underline{\text { Les suggestions de la recherche }}$}

En basant sur les résultats de la recherche actuelle, on peut proposer les thèmes de recherche suivants

1- L'effet de l'utilisation des activités langagières pour développer quelques habiletés de la cohérence textuelle chez les étudiants du niveau universitaire.

2-Programme proposé basé sur la bande dessinée pour développer quelques habilités de l'interprétation des images séquentielles et la production écrite de la langue française chez les étudiants au niveau universitaire.

\section{Bibliographie}

\section{Références}

\section{Thèses}

1) Abel-Ghany, Ch. (2003) : L'efficacité d'une stratégies proposée établie sur le style de l'exercice guidé et la résolution des problèmes dans le développement de quelques compétences de l'expression écrite libre chez les étudiants de quatrième année de section de français à la faculté de pédagogie de Tanta. Thèse de Magistère, Faculté de pédagogie, Univ. de Tanta.

2) Carol Beaupré (1988) : cohésion et cohérence dans l'écriture d'un récit interactif a l'ordinateur, université de Montréal, thèse de doctorat en sciences de l'éducation.

3) El-Kouche, A. (2004) : L'Efficacité de l'Emploi de l'Approche Par Le Processus sur le développement des Habiletés d'Ecriture et les Attitudes des étudiants du Département de la Langue Française. Thèse de Doctorat, Univ. de Caen, Basse-Normandie.

4) Emmanuelle BEAULIEU HANDFIELD (2008) : Rupture des cohérences dans les écrits de étudiants universitaires, mémoire présente de la maitrise en linguistique, université de Québec à Montréal, juillet 2008.

5) Sabri, N. (2005) : Efficacité de L'Approche Constructiviste sur Le développement de Quelques Compétences de L'Expression Ecrite Fonctionnelle chez Les Etudiants Maîtres du Département de Français. Thèse de Magistère, Faculté de Jeunes-Filles, Univ d'Ain-Chams.

\section{$\underline{\text { Livres et périodiques }}$}


1) Antonin Brunet, (2020) : "Quel corpus pour l'identification des compétences des apprenants de niveaux intermédiaire et avancé ? Les cas de la cohérence et de la cohésion », Lidil [En ligne], 61 | 2020.

2) Bouchard, R. 2000. «Ecriture en L2, Registres, Pré constructions. Des questions pour la seule didactique des langues? Et/ou pour la RAL? ». In Etudes de linguistique appliqué $\mathrm{n}^{\circ} 120$. Paris, Didier Erudition, pp. 475- 486.

3) Catherine Muller, (2013) : L'image en didactique des langues et des cultures : une thématique de recherche ancienne remise au goût du jour,

Revue du Gerflint, Synergies Portugal n 2 - 2014 p. 128.

4) CHALANE Jugurtha et BRAHMI Fatsah (2016-2017) : Enseignement de la cohésion textuelle au secondaire, mémoire de master, faculté de lettres et des langues, département de français, université de Abderrahmane Mira, Bejaia

5) Claire Pecquery (2013) : fiche exercice, au programme scolaire de $\mathrm{CP}$,La règle, le principe : Décrire une image.

6) Conseil de l'Europe. (2001). Un cadre européen commun de référence pour les langues : apprendre, enseigner, évaluer. Paris : Didier.

7) Daniel Maja (2004 ): L'illustration jeunesse, éditions du Sorbier

6) Fontenay le Comte, et Luçon (2013 /2014) : plaisir de écrire cycle 2 et 3 Dispositif d'impulsion des pratiques de production d'écrits dans les disciplines, Ecrire à partir d'images séquentielles au CP

7) Helmi, Z. (2000) : Canevas de Simulation Globale en vue du Développement de la Créativité au Niveau Universitaire. Revue de Faculté de Jeunes-Filles, Univ d'AinChams, Vol : 1 .

8) HAROLLES, J.M., (1988) : «Les études sur la cohérence, la cohésion et la connexité textuelles depuis la fin des années 1960. », Modèles linguistiques, 10 (2), p. 45-66.

9) Jacqueline Lemieux, Helene Fortier, pierre Rossignol (1987): « le français écrit par la cohérence du texte », campus Notre Dame de Foy

10) M. Martineau (2012) : enseignement d'exploration littérature et société tag, Fiche méthode $\mathrm{n}^{\circ} 3$ : Comment analyser une photographie?

11) Mohammed Alkhatib, (2012) : la cohérence, la cohésion textuelles problème linguistique ou pédagogique, université de Nizwa, sultanat Oman

12) Proje-cretien Joëlle, Alves Christian (1989): les images séquentielles : De la lecture de images à la naissance du récit in : Spirale revue de recherches en éducation, no 2 ,1989. 
13) Saif Elislam lofty Abdul Hakeem (2020) : Programme d'entrainement proposé pour développer la capacité des apprenants de FLE à analyser et à interpréter les images fixes à la lueur de l'approche sémiologique Revue de Faculté de Jeunes-Filles, Univ d'Ain-Chams, Vol :21 no 21.

14) Vandendrope Christian (1995:9) : « au-delà de la phrase : la grammaire du texte » pour un nouvel enseignement de la grammaire, coll. sous la dire .de Suzanne-G Chartrand Montréal les Editions logique, 1995p83-105

15) Van Dijk Teun A (1984): « le texte »dans dictionnaire des littératures de langue française Tom III Paris Bordas p 2282.

\section{$\underline{\text { Dictionnaires }}$}

1) «Dictionnaire du français » (2001) : référence apprentissage cycle secondaire première langue CLE international, ministère de l'éducation et de l'enseignement

2) «Lexique de termes pédagogiques couramment utilisés dans le monde éducatif et de l'enseignement » (2006) : Arts plastiques / Académie de Lille / Septembre 2006.

\section{$\underline{\text { Références sites Internet }}$}

1) CHAROLLES Michel. 2011: «Cohérence et cohésion du discours ». (En ligne), consulté le 29/12/2019,

URL : https://hal.archives-ouvertes.fr/hal-00665838/document

2) eduscol. Éducation. fr /ressources 2016 ,

Ministère d'éducation national de l'enseignement supérieur et de la recherche

3) B. BOISHARDY (2008): CP Angers 3 (49) - pour les Circonscriptions de St Philbert de Grand Lieu (44) et d'Angers 3 (49) L. Pallard (49), recto-verso n56: les images séquentielles

(En ligne), consulté le 7/11/2020,

URL : https://www.pedagogie.ac-nantes.fr/francais/documents/recto-verso-n-56-lesimages-sequentielles-679437.kjsp?RH=PEDA. 
استخدام الصور المتتابعة لتنمية مهارتى التأويل والتلاحم التصى واثره على التعبيز الكتابى لاى التى الطلاب المعلمين يشعبة اللغة الفرنسية بكلية التربية جامعة ولئة المني

\section{د/ سيف الإسلام لطفى عبد الحكيم خليفة}

مدرس بقسم المناهج وطرق التدريس، كلية التربية، جامعة المنيا

المستخلخص:

يعد التعبير الكتابى أداة المعلم فى تعليم المتعلم وأداة المتعلم فى توضيح ما تعلمه و ابر ازه للاخرين و الكثف عن مدار مدى فهمه

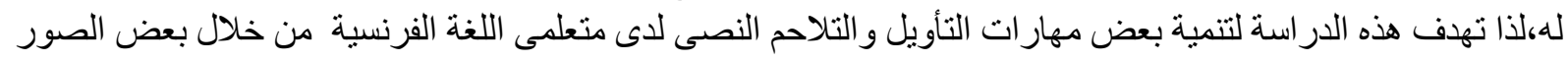

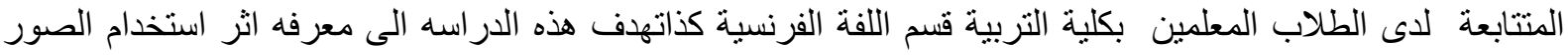

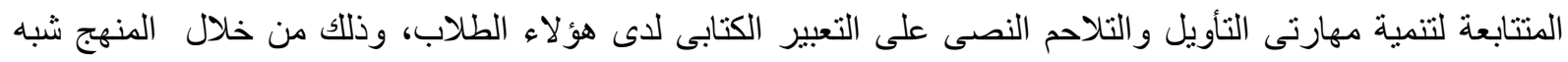

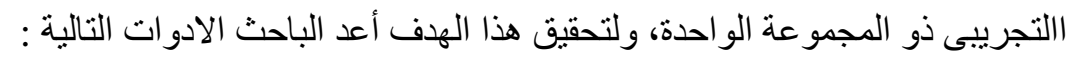

- ق قائمة بمهارات تأويل الصورر المتتابعه اللازمة للطلاب المعلمين بكلبة التربية شعبة اللفة الفرنسية بكلية التربية جامعة المنيا.

قائمة بمهار اتربة التلاحم النصى اللازمة للطلاب المعلمين بكلية التربية شعبة اللفة الفرنسية بكلية التربية جامعة المنيا.

قائمة بمهارات التعبير الكتابى المفترض اكسابها للطلاب المعلمين بشكل سليم والتى تتم من خلال استخدام

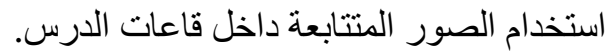

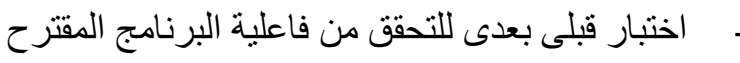

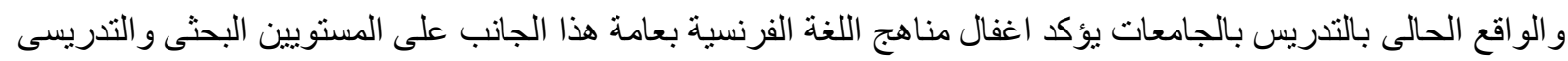

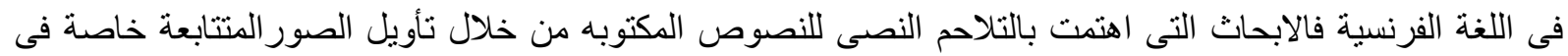
اللغة الفرنسية بمر احل التعليم الجامعى تكاد تكون محدودة على حلى التح علم الباحث فى هذا المجال.

لذا يهدف هذا البحث لتنمية مهارنى تأويل الصور المتتابعة و التلاحم النصى ل لادى متعلمى اللغة الفرنسية ، وقد

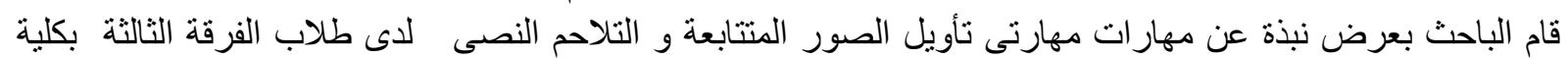

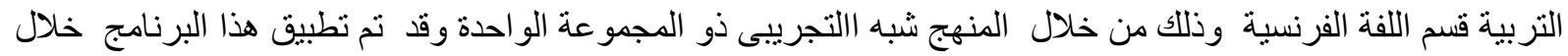

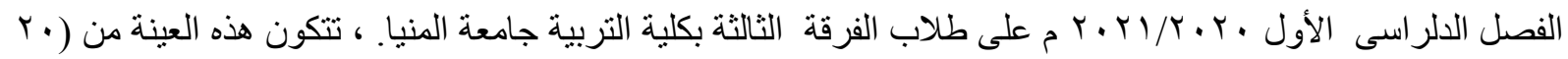

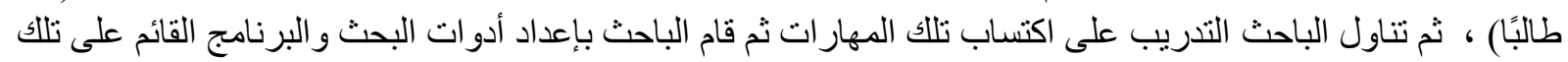

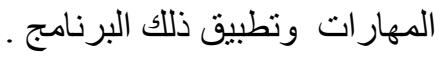

وقد توصلت الدر اسة الى وجود فرق دو دلالة احصائية بين متوسط درجات الطلاب في مهارتى تأويل الصور

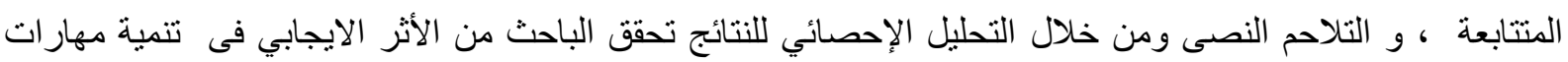

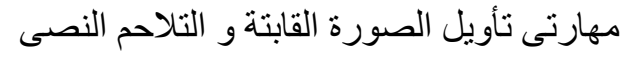

الكلمات المفتاحية: الصور المتتابعة ـ التأويل ـ التلاحم النصى ـ التعبير الكتابى

Received on: 20 /8 / 2021 - Accepted for publication on : 6/10 /2021- E-published on: 9/ 2021 